\title{
Alkylating HIV-1 Nef - a potential way of HIV intervention
}

Yong-Jiu Jin ${ }^{*}$, Xiaoping Zhang ${ }^{2}$, Catherine Yi Cai ${ }^{1}$, Steven J Burakoff ${ }^{1,3}$

\begin{abstract}
Background: Nef is a $27 \mathrm{KDa}$ HIV-1 accessory protein. It downregulates CD4 from infected cell surface, a mechanism critical for efficient viral replication and pathogenicity. Agents that antagonize the Nef-mediated CD4 downregulation may offer a new class of drug to combat HIV infection and disease. TPCK ( $\mathrm{N}-\alpha-\mathrm{p}$-tosyl-Lphenylalanine chloromethyl ketone) and TLCK (N- $\alpha$-p-tosyl-L-lysine chloromethyl ketone) are alkylation reagents that chemically modify the side chain of His or Cys residues in a protein. In search of chemicals that inhibit Nef function, we discovered that TPCK and TLCK alkylated HIV Nef.

Methods: Nef modification by TPCK was demonstrated on reducing SDS-PAGE. The specific cysteine residues modified were determined by site-directed mutagenesis and mass spectrometry (MS). The effect of TPCK modification on Nef-CD4 interaction was studied using fluorescence titration of a synthetic CD4 tail peptide with recombinant Nef-His protein. The conformational change of Nef-His protein upon TPCK-modification was monitored using CD spectrometry

Results: Incubation of Nef-transfected T cells, or recombinant Nef-His protein, with TPCK resulted in mobility shift of Nef on SDS-PAGE. Mutagenesis analysis indicated that the modification occurred at Cys55 and Cys206 in Nef. Mass spectrometry demonstrated that the modification was a covalent attachment (alkylation) of TPCK at Cys55 and Cys206. Cys55 is next to the CD4 binding motif $\left(\mathrm{A}_{56} \mathrm{~W}_{57} \mathrm{~L}_{58}\right)$ in Nef required for Nef-mediated CD4 downregulation and for AIDS development. This implies that the addition of a bulky TPCK molecule to Nef at Cys55 would impair Nef function and reduce HIV pathogenicity. As expected, Cys55 modification reduced the strength of the interaction between Nef-His and CD4 tail peptide by $50 \%$.
\end{abstract}

Conclusions: Our data suggest that this Cys55-specific alkylation mechanism may be exploited to develop a new class of anti HIV drugs.

\section{Background}

Nef proteins of primate lentiviruses, HIV-1, HIV-2 and SIV, are abundantly expressed in the early phase of HIV-1 infection and play a crucial role in the pathogenicity of HIV-1 and the development of AIDS [1-8]. One prominent piece of evidence is that HIV-1 strains isolated from some long-term survivors carried deletions or truncations of nef exclusively $[9,10]$. The pathological roles of Nef in the development of AIDS have been attributed to several Nef biological activities, including downregulation of the viral primary receptor CD4 [11] and downregulation of the cell surface expression of class-I major histocompatibility complex (MHC-I)

\footnotetext{
* Correspondence: Yong-Jiu.Jin@mssm.edu
'Department of Oncological Sciences, Mount Sinai School of Medicine, New

* Correspondence: Yong-Jiu.Jin@mssm.edu
'Department of Oncological Sciences, Mount Sinai School of Medicine, New York, NY 10029, USA
}

(c) 2010 Jin et al; licensee BioMed Central Ltd. This is an Open Access article distributed under the terms of the Creative Commons Attribution License (http://creativecommons.org/licenses/by/2.0), which permits unrestricted use, distribution, and reproduction in any medium, provided the original work is properly cited. in favor the viral replication by engaging several signaling molecules, such as Vav, Pak2, ASK1 and Src family kinases [14-18] (for reviews, see [19,20]). Nef has no known catalytic activity; it acts essentially as a connector to link CD4, MHC-I, and possibly some other target molecules to adaptor protein (AP) complexes AP-1, AP2 or AP-3, responsible for the endocytosis and subsequent lysosomal degradation of Nef's targets. We found that Nef-mediated CD4 downregulation is AP-2 dependent and required an ubiquitinated lysine residue K144 in HIV-1 Nef [21,22]. The structure of HIV-1 Nef has been established by NMR and X-ray crystallography [23-25] (see [26] for a review). HIV-1 Nef protein consists of a conserved core domain of about 120 residues and two flexible regions - the $\mathrm{N}$-terminus 68 amino 
acids flexible arm and a 32 amino acid loop structure (V148-L181) located in the C-terminal region. The HIV protease cleavage site $\mathrm{C}_{55} \mathrm{AW}{ }_{57} \mathrm{LEA}$ [27] and CD4 binding motif $\left(\mathrm{A}_{56} \mathrm{~W}_{57} \mathrm{~L}_{58}\right)$ [28] are located in Nef N-terminal region. Nef is myristoylated at a Gly residue (G2) in the $\mathrm{N}$-terminus, which mediates the membrane association of Nef [29]. The core domain is a $\alpha-\beta$ globular structure responsible for Nef binding to SH3 domaincontaining proteins $[16,30,31]$. The loop in the C-terminal region contains the dileucine motif $\operatorname{ExxxLL}_{160}$, which interacts with adaptor protein complexes AP-1, 2, 3 [32-34].

TPCK (N- $\alpha$-p-tosyl-L-phenylalanine chloromethyl ketone) and TLCK (N- $\alpha$-p-tosyl-L-lysine chloromethyl ketone) are alkylation reagents that can chemically modify side chains of specific His or Cys residues in some proteins. It is known that TPCK modifies His in the reactive center of serine protease chymotrypsin and trypsin, resulting in enzymatic inhibition $\left(E_{50}\right.$ of 20 $\mu \mathrm{M}$ and $80 \mu \mathrm{M}$, respectively) $[35,36]$. TPCK and TLCK also alkylate the sulfhydryl group of the Cys residue in several other proteins, including protein kinase $\mathrm{C}$ [37,38], cAMP-dependent kinase [39,40], HPV-18 E7 [41] and human ETS 1 oncoprotein [42]. Alkylation of Cys side chains makes HPV-18 E7 [41] and human ETS 1 oncoprotein [42] migrate faster on SDS-PAGE.

\section{Methods}

Cells, antibodies and chemicals

SV40 T antigen-transfected human leukemic Jurkat T cells (JTAg) were cultured in RPMI medium supplemented with 10\% FCS. For transient expression, plasmid DNA was transfected into the cells using Lipofectamine $2000^{\text {tm }}$ (Invitrogen). Anti-HIV-1 Nef rabbit serum was obtained from NIH AIDS Research and Reference Reagent Program. N-tosyl-L-phenylalanine chloromethyl ketone (TPCK), NA-p-tosyl-L-lysine chloromethyl ketone (TLCK) and N-CBZ-Phe-Ala fluoromethyl ketone (Z-FA-FMK) were purchased from Sigma (Saint Louis, MO).

\section{Plasmids}

HIV-1 Nef (NA7)-GFP plasmid kindly provided by Dr. J. Skowronski was subcloned into pcDNA3 to express untagged wt Nef (NA7). Nef $\left(\mathrm{G}_{2} \mathrm{G}_{3} / \mathrm{AA}\right)$ mutant was generated by PCR mutagenesis as described before [43]. Nef (NL4-3) was PCR subcloned into pcDNA3 vector with the template of HIV-1 (NL4-3) provirion from NIH AIDS Research and Reference Reagent Program. Nef Cys-to-Ala mutants C55/A, C142/A, C206/A, C55\&206/ A, C55\&142/A, C142\&206/A and C55\&C142\&C206/A (Cys free) were generated by PCR mutagenesis with wt Nef (NA7) plasmid template using Multi-Quick Change Mutagenesis kit (Stratagene). For E. coli cell expression, wt Nef and Nef mutants were subcloned into pET-30a $(+)$ vector (Novagen) at Nde I/Not I sites. All mutations generated in this study were confirmed by DNA sequencing.

\section{Analysis of Nef modification in TPCK- or TLCK-treated JTAg cells}

Analysis was performed using Nef (NA7) transfected JTAg cells unless otherwise specified. Cells were transfected with Nef plasmid DNA for 16-20 h and treated with TPCK/TLCK $(10 \mu \mathrm{g} / \mathrm{ml})$ for $30 \mathrm{~min}$. Cells $(2 \times$ $10^{5}$ ) were boiled in $25 \mu \mathrm{l} 2 \times$ SDS sample buffer and loaded to $11 \%$ reducing SDS-PAGE. Nef protein was detected by immunoblotting with polyclonal anti-Nef (1:10,000 dilution) at $\mathrm{RT}$ for $2 \mathrm{~h}$ or at $4{ }^{\circ} \mathrm{C}$ overnight, followed by ECL anti-rabbit Ab $(1: 10,000)$ at RT for $1 \mathrm{~h}$.

\section{Nef-His protein preparation and in vitro modification}

Plasmid encoding Nef-His in pET-30a (+) vector was transformed into E. coli BL21 cells. The transformed cells were grown in $\mathrm{LB}$ medium at $37^{\circ} \mathrm{C}$ for $16 \mathrm{~h}, 1: 10$ diluted with fresh LB, and induced with IPTG $(1 \mathrm{mM})$ for 3 hours. Four hundred $\mathrm{ml}$ of cells were pelleted, washed with PBS and lysed by sonication. Nef-His protein was isolated with a HisTrap column (Amersham Biosciences) or using Ni-NTA agarose beads (QIAGEN). The beads were washed three times in $20 \mathrm{mM}$ Imidazole/PBS. Nef-His was eluted with $250 \mathrm{mM}$ Imidazole, adjusted with PBS to the concentration of UV absorbance $\left(\mathrm{A}_{280}\right)=1.0$, and kept at $-20^{\circ} \mathrm{C}$ before use. For in vitro modification, freshly prepared $\mathrm{Nef}-\mathrm{His}$ was incubated with TPCK $(10 \mu \mathrm{g} / \mathrm{ml})$ at RT for $30 \mathrm{~min}$. Twenty $\mu \mathrm{l}$ of samples was resolved by SDS-PAGE. The gels were stained with Coomassie Blue or immunoblotted with anti-Nef.

\section{Mass spectrometry}

Nef-His protein was in vitro modified with TPCK as described above. The completion of the modification was confirmed by SDS-PAGE. Fifty $\mu \mathrm{g}$ of the un-modified and TPCK-modified Nef-His proteins were analyzed by MS to determine the molecular weight. For trypsindigestion, $20 \mu \mathrm{g}$ of Nef-His was denatured in $0.1 \mathrm{M}$ ammonium bicarbonate at $55^{\circ} \mathrm{C}$ for $30 \mathrm{~min}$ and then digested at $37^{\circ} \mathrm{C}$ with trypsin at $1: 100(\mathrm{w} / \mathrm{w})$. The samples were subjected to mass spectrometry (MALDI-ToF) at the NYU medical school service center using MS spectrometer Micromass (Waters).

\section{Fluorescence titration of CD4 tail peptide with HIV-1 Nef}

Fluorescein-labeled CD4 tail peptide (FluoresceinQAERMSQIKRLLSEKKT, residue 403-419) was synthesized by Sigma. Fluorescence emission was recorded with a FluoroMax-2 fluorescence spectrometer 
(excitation at $492 \mathrm{~nm}$; emission at $516 \mathrm{~nm}$ ). CD4 tail peptide of $1.0 \mu \mathrm{M}$ in PBS was analyzed in a stirred cuvette at $25^{\circ} \mathrm{C}$. Data were collected after $30 \mathrm{~min}$ incubation with Nef-His. Controls incubated with PBS did not show reduction in fluorescence. Experimental signals were expressed as the percentage of fluorescence reduction averaged from three independent measurements. The signals were plotted against total Nef concentration.

\section{CD spectrum of Nef-His}

Nef-His protein of $100 \mu \mathrm{M}(\sim 2.5 \mathrm{mg} / \mathrm{ml})$ in PBS, $\mathrm{pH}$ 7.4, was subjected to $\mathrm{CD}$ spectrometry analysis. Far-UV $\mathrm{CD}$ measurement at $20^{\circ} \mathrm{C}$ was made on an Aviv $202 \mathrm{CD}$ spectrometer (Lakewood, NJ) in the department of chemistry of NYU. Data were the average of 4-6 accumulations, using scanning wavelength of 260-195 nm, speed of $20 \mathrm{~nm} / \mathrm{min}$, bandwidth of $1 \mathrm{~nm}$, and response time of $0.5 \mathrm{~s}$. Data were plotted using the SigmaPlot software.

\section{Results}

TPCK and TLCK modified HIV-1 Nef expressed in culture T cells

JTAg cells were transfected with plasmids encoding HIV-1 Nef NA7 or NL4-3 and treated with one of the three alkylating reagents, TPCK, TLCK, or Z-FA-FMK. Fig.1A shows that TPCK- or TLCK-treatment altered the mobility of both Nef NA7 and Nef NL4-3 proteins on SDS-PAGE. About 20-30\% Nef proteins migrated faster with the treatments (indicated by the letter " $F$ ") whereas a small fraction of Nef protein migrated slower (indicated by the letter "S"), which was more noticeable with TLCK than with TPCK. In contrast, treatment with similar doses of Z-FA-FMK did not affect the mobility of Nef protein on SDS-PAGE (Fig.1A). TPCK and TLCK contain chloromethyl ketone whereas Z-FA-FMK contains fluoromethyl ketone (boxed in Fig.1B). The results suggest that Nef proteins may be specifically modified by TPCK and TLCK. TPCK/TLCK at a dose of $1-2 \mu \mathrm{g} / \mathrm{ml}(\sim 5-10 \mu \mathrm{M})$ was effective in the modification. This dose is lower than the EC50 of TPCK (20 $\mu \mathrm{M})$ and TLCK $(80 \mu \mathrm{M})$ in their serine protease inhibition (sigma product information), suggesting a higher reaction specificity of TPCK/TLCK with Nef than with serine proteases. The modification is independent of Nef myristoylation and membrane association since the myristoylation-defective $\operatorname{Nef}\left(\mathrm{G}_{2} \mathrm{G}_{3} / \mathrm{AA}\right)$ mutant was also modified with TPCK (Fig.1A bottom).

\section{TPCK modified Nef at Cys55 and Cys206}

It was reported that TPCK-treatment altered the mobility of HPV-18 E7 and human ETS 1 oncoprotein on SDS-PAGE as a result of Cys alkylation [41,42]. HIV-1 Nef contains two conserved Cys residues (Cys55 and

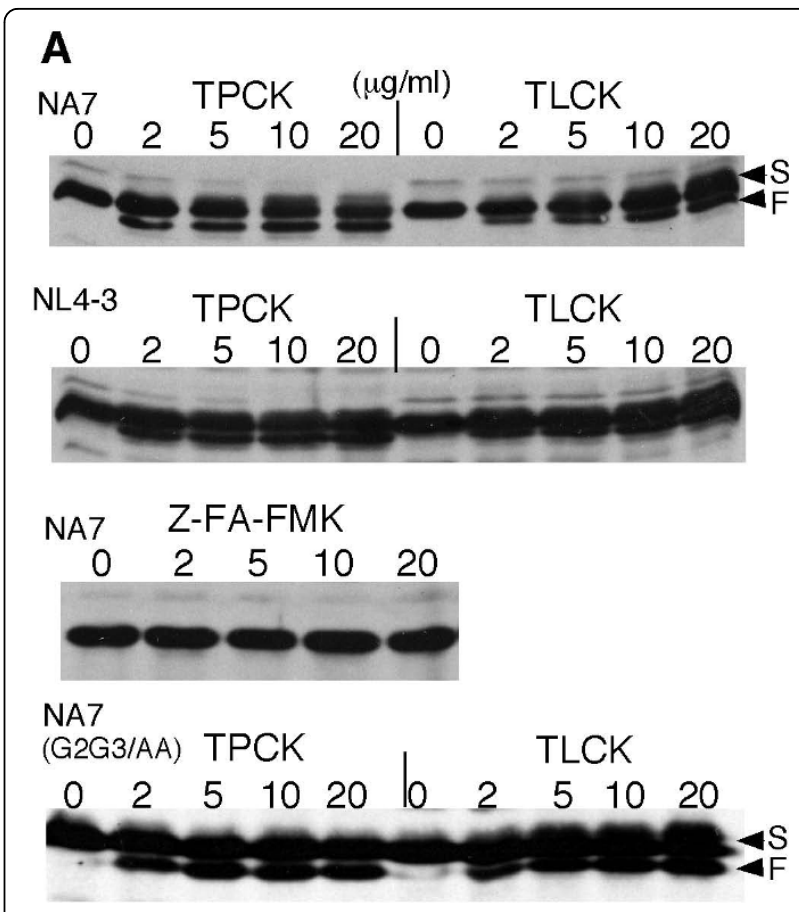

B
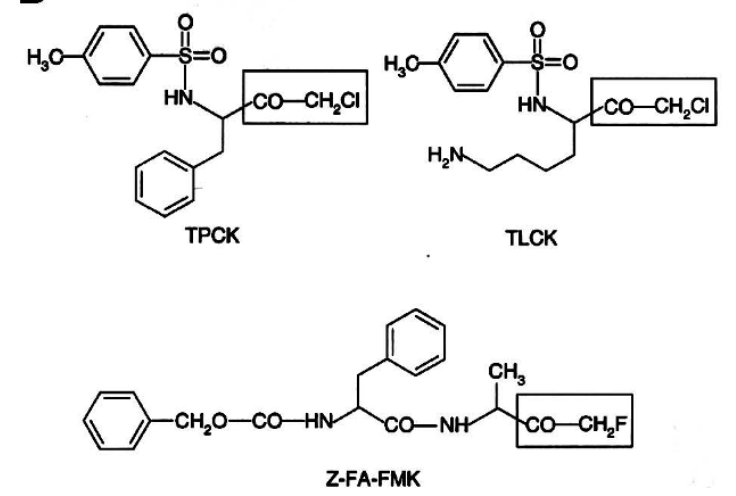

Figure 1 Treatment of Nef transfected T cells with TPCK or TLCK altered the mobility of Nef on SDS-PAGE. (A) Anti-Nef immunoblotting of Nef proteins from TPCK, TLCK or Z-FA-FMK treated cells. JTAg cells were transfected with Nef NA7 (upper panel), Nef NL4-3 (middle panel) or NA7 $\left(G_{2} G_{3} / A A\right)$, treated with TPCK, TLCK or Z-FA-FMK for $30 \mathrm{~min}$ as indicated. The whole cell lysates were immunoblotted with anti-Nef. Arrows indicate the faster (F) or slower (S) migrated Nef proteins. (B) Structures of TPCK, TLCK and Z-FA-FMK. The boxed atoms are the alkylating groups reacting with specific His or Cys residues in substrate proteins.

Cys142) and a partially conserved C-terminal Cys (Cys206) [44]. To find out whether Nef was also modified at Cys residues, we examined the mobility of TPCK-treated Nef Cys mutants on SDS-PAGE. Fig. 2 shows that TPCK-treatment did not cause any mobility shift of Cys-free Nef mutant (upper left panel), suggesting that Cys residues were the residues to be modified. Double Cys mutant C55\&206/A showed no mobility 


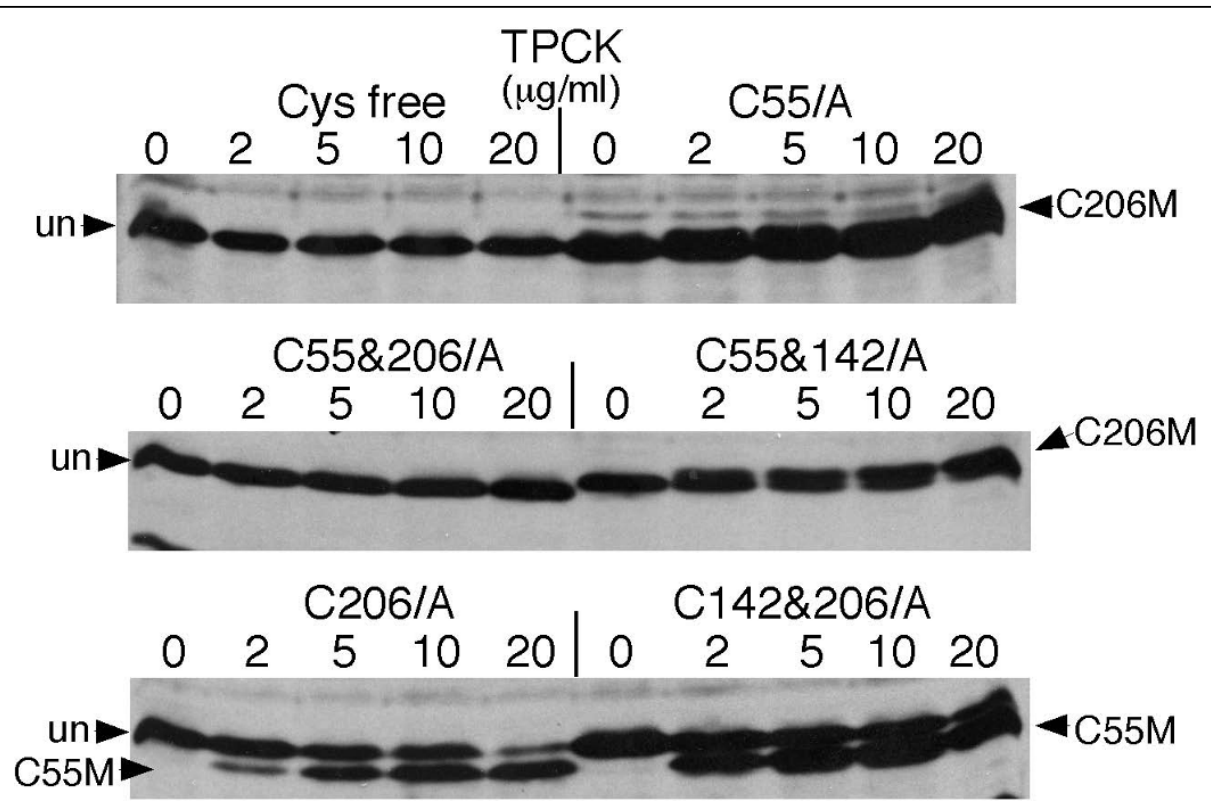

Figure 2 TPCK-modification of Nef mutants with Cys55, Cys142, and/or Cys206 substituted with Ala. Plasmid encoding Nef mutant C55/ A, C206/A, C142\&206/A, C55\&206/A, C55\&142/A or C55\&C142\&c206/A (Cys free) were transfected into JTAg cells. TPCK modification was determined as described in Fig. 1. Arrows indicate the Cys206-modified (C206M), Cys55-modified (C55M) and the un-modified Nef (un) proteins.

shift either (middle left panel), suggesting that Cys55 \&/or Cys206, but not Cys142, were the residues modified. Further more, single cysteine mutant C55/A migrated slower on SDS-PAGE, indicating that the modification at Cys206 resulted in a slow migration form of Nef (C206M) whereas Nef mutant C206/A migrated faster on SDS-PAGE (bottom panels), indicating that the modification at Cys55 resulted in a faster migration form (C55M). The migration patterns of Nef mutant C142\&206/A (Cys55 modified) and C55\&142/A (Cys206 modified) were the same as that of C206/A and C55/A. Taken together, the mutagenesis data suggest that Cys55 and Cys 206 but not C142 and His residues are modified by TPCK. This conclusion is directly proved by the following MS analysis.

\section{TPCK modified recombinant Nef-His protein in vitro and} the modification appeared to be dependent on Nef conformation

Next we asked whether TPCK-modification of Nef is a direct chemical reaction. The E. coli expressed, isolated Nef-His protein was incubated with TPCK in PBS. Fig. 3A shows that Nef-His protein was modified with TPCK in vitro, resulting in a faster mobility shift on SDSPAGE. The results indicated that the modification is a direct chemical reaction between Nef and TPCK. Notably, we found that the freshly prepared Nef-His protein was modified efficiently, with a yield of $\sim 80$ to $95 \%$. But the modification yield was greatly decreased if Nef-His protein in PBS had been kept at $4^{\circ} \mathrm{C}$ for 1-2 days before

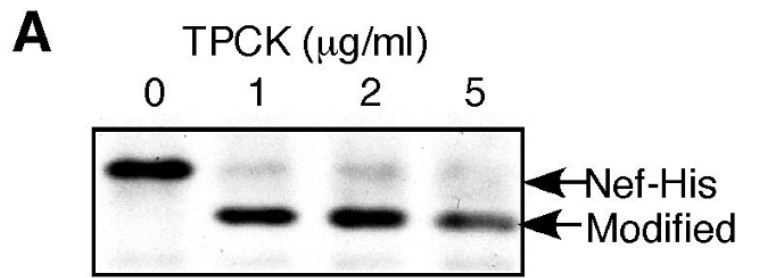

B

Modification after pre-incubation in PBS

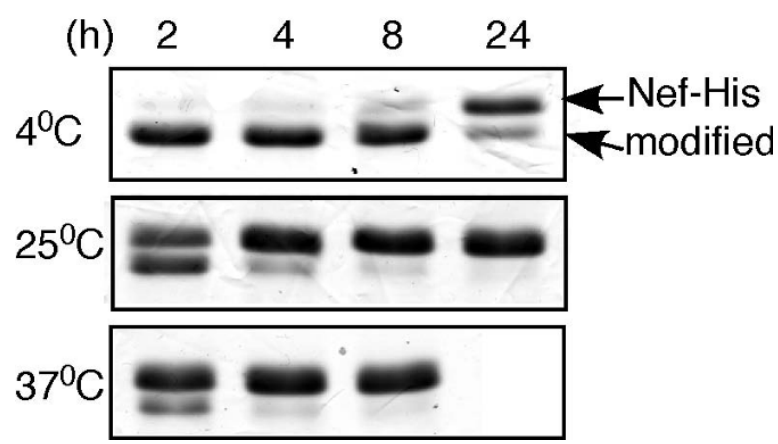

Figure $\mathbf{3}$ In vitro modification of Nef-His protein by TPCK E. coliexpressed Nef-His protein was isolated using Ni-beads as described in Methods. Twenty $\mu \mathrm{l}$ of freshly prepared Nef-His protein at the concentration of $\sim 0.5 \mu \mathrm{g} / \mu \mathrm{l}$ was incubated with TPCK in PBS at RT for $30 \mathrm{~min}$ and then resolved by SDS-PAGE. The gels were stained with Coomassie Blue. (A) TPCK-modification of the freshly prepared Nef-His protein at different TPCK concentrations. (B) TPCK-

modification of the Nef-His protein pre-incubated in PBS at different temperatures for different length of times. 
incubation with TPCK. At higher temperature of $25^{\circ} \mathrm{C}$ or $37^{\circ} \mathrm{C}$, an 8 or $4 \mathrm{~h}$ pre-incubation was enough to almost abrogate the modification (Fig. 3B). As shown in the figure, Nef-His was not degraded during the incubation. Since it is known that the isolated recombinant Nef protein is unstable and undergo conformational change \&/or aggregation at higher concentrations [24], the results suggested that a potential conformational change of Nef may affect its modification with TPCK. It is possible that the overexpressed Nef protein in culture cells also undergoes conformational change \&/or aggregation, which could explain why Nef was only partial modified with TPCK (Fig.1). Supporting this notion, we observed that alkylation efficiency of Nef in culture cells was reduced when the new Nef protein synthesis was stopped by addition of cycloheximide and MG132 in cell culture for several hours (data not shown).

\section{MS analysis proved that TPCK was covalently bound to Cys 55 and Cys 206 but not to His residues}

To prove that TPCK is covalently bound to Cys55 and Cys206 and to rule out that TPCK may modify some other Nef residues unaffecting Nef's mobility, we analyzed the TPCK-modification of Nef-His by mass spectrometry (MS). Fig. 4A shows the molecular weight of untreated and TPCK-treated Nef-His determined by MS. The peak of untreated Nef-His is 24386 Dalton (TPCK-) whereas TPCK-treated Nef-His is 25011 Dalton (TPCK+). The 631 Dalton difference equals the molecular weight of two TPCK molecules $(2 \times 352$ Dalton) minus two $\mathrm{HCl}$ molecules $(2 \times 36.5$ Dalton), indicating that each Nef molecule was covalently bound with two TPCK molecules. To prove that TPCK was bound to Cys55 and Cys206, we did a tryptic mapping (Fig. 4B). Amino acid sequence of Nef-His predicts that tryptic peptide of $1430 \mathrm{Da}$ (P1430) contains Cys206, tryptic peptide of $4787 \mathrm{Da}$ (P4787) contains Cys55, and tryptic peptide of $1263 \mathrm{Da}$ (P1263) contains Cys142. All these peptides were detected (indicated by arrows) in untreated Nef-His. With TPCK-modification, P1430 and P4787 were converted to P1745 and P5100. TPCK-treatment did not affect P1263, indicating that Cys142 is not alkylated. Note, we have to use a high sensitivity scale for detection of P4787 (up right panel) due to its low UV absorbance. With the attachment of TPCK (N- $\alpha-\mathrm{p}-$ tosyl-L-phenylalanine chloromethyl ketone) - a highly UV detectable chemical, P5100 (4787+TPCK) and P1745 (1430+TPCK) (bottom panels) exhibited a much higher UV absorbance. We also sequenced the tryptic peptide P1715 that contains the very C-terminal His-tag and Cys206 (Fig. 4C). The results showed that none of the His residues in His-tag was alkylated, whereas Cys206 was. Residue B of the peptide (Cys206, circled in Fig. 4C) had a molecular weight of $418 \mathrm{Da}$, exactly equal to that of a one TPCK-alkylated Cys. Thus, the collective MS data proved that TPCK alkylates Cys55 and Cys206 but not Cys142 or any His residues.

\section{TPCK alkylation at Cys55 severely impaired Nef's interaction with CD4 tail peptide}

Cys55 is next to Nef motif A56W57L58, a site implicated in the interaction of Nef with CD4, Nef-mediated CD4 downregulation and the onset of AIDS $[9,10,28]$. To ask whether the attachment of a bulky TPCK molecule to Cys55 affects Nef-CD4 interaction, we performed an in vitro $\mathrm{CD} 4-\mathrm{Nef}$ binding assay following a published protocol [45]. In the assay, a fluorescein-labeled 17 amino acid CD4 tail peptide was incubated with Nef-His or TPCK modified Nef-His proteins at increase concentrations. Quenching of the fluorescence emission from the label CD4 peptide by Nef-His proteins was measured as the results of CD4-Nef interaction [45]. Fig. 5 (left panel) compares the titration curve of the unmodified wt Nef-His with that of TPCK-modified Nef-His. The results showed that the fluorescence emission was quenched by $11.6 \%$ with unmodified Nef-His protein $(10 \mu \mathrm{M})$ whereas was quenched by $4.9 \%$ with TPCKmodified Nef-His, indicating that TPCK-modification resulted in more than $50 \%$ of decrease in the strength of Nef-CD4 interaction. To confirm that the effects are C55 modification specific, we also compared the titration curve of the unmodified Nef mutant (C55/A)-His with that of TPCK-treated Nef (C55/A)-His. Fig. 5 (right panel) shows that the titration curves of the untreated and treated (C55/A)-His were quite similar. At $10 \mu \mathrm{M}$ concentration, the level of quench was $10.6 \%$ and $9.8 \%$ for untreated and treated, respectively, confirming that the effects are depended on modification of Nef C55. We concluded that the alkylation at Cys 55 will greatly impair Nef-CD4 interaction and, therefore, would weaken HIV-1's pathogenicity. In addition, the fluorescence reduction by wt Nef-His was $11.6 \%$ compared with $10.6 \%$ by Nef (C55/A)-His, suggesting that C55A mutation itself may have a weak effects on Nef-CD4 interaction.

\section{CD spectrometry data indicated a moderate Nef conformational change after TPCK alkylation}

To ask whether alkylation alters the solution structure of Nef, we compared the CD spectrometry of Nef-His proteins unmodified or modified with TPCK (Fig. 6). The CD spectrometry showed that Nef has an overall $\alpha-\beta$ structure with an absorbance of $\alpha$-helix at $208 \mathrm{~nm}$ and absorbance of $\beta$-sheet at $216-220 \mathrm{~nm}$. TPCK alkylation did not result in a shift of the absorbance wavelength $(\mathrm{nm})$, suggesting that there was no global change in the overall $\alpha-\beta$ structure. However, the $\alpha$-helix absorbance at $\sim 208 \mathrm{~nm}$ apparently became weaker, suggesting a less 


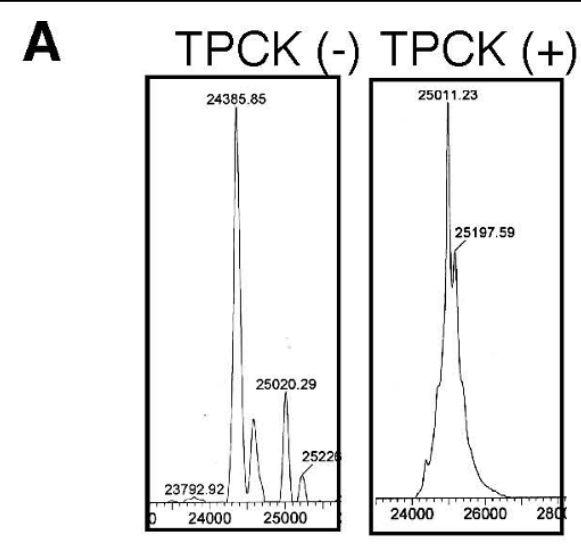

B
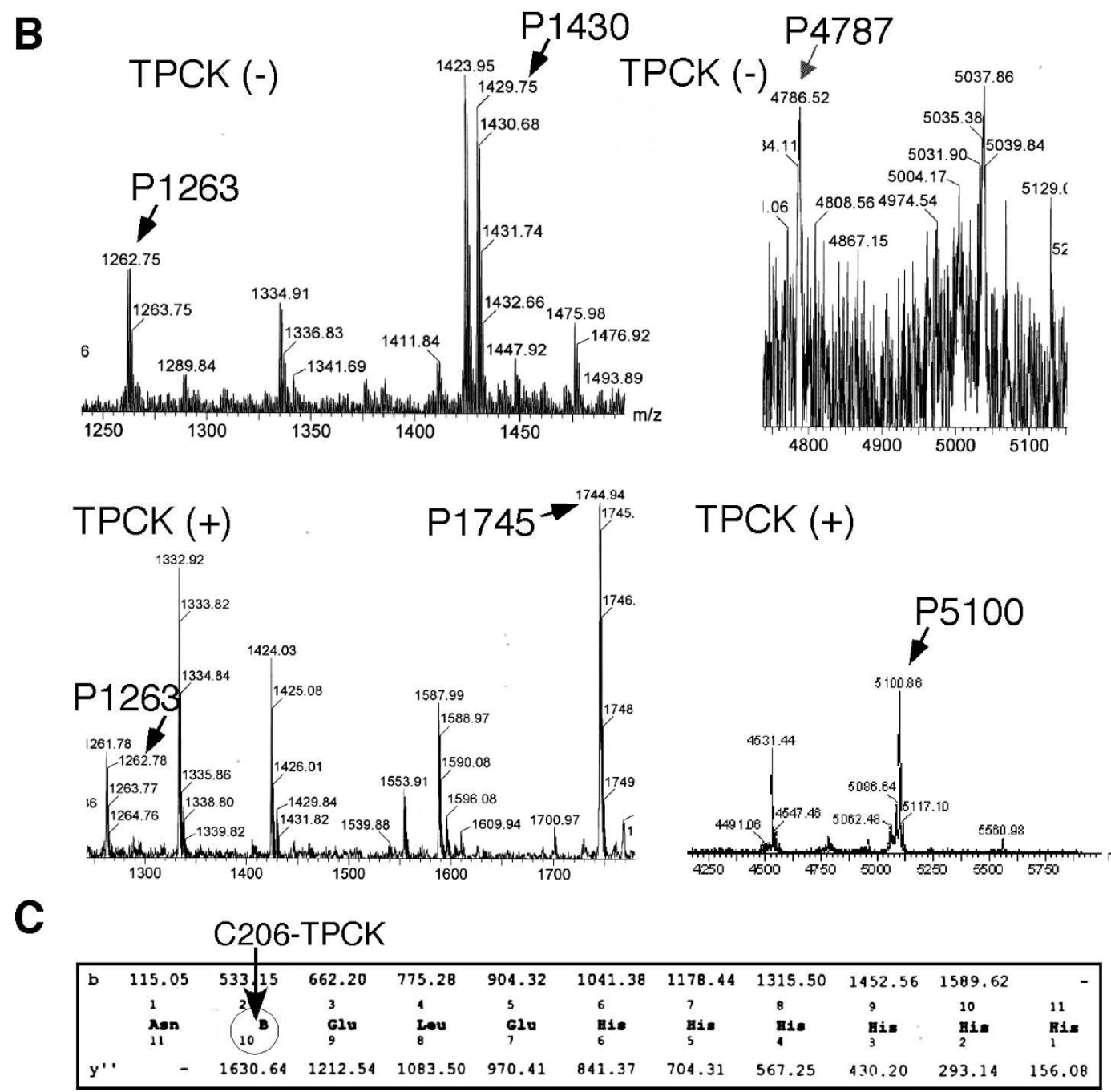

Figure 4 Mass Spectrometry (MS) of the unmodified and TPCK-modified Nef-His proteins. (A) MS- determination of the molecular weight of the unmodified (TPCK-) and modified (TPCK+) Nef-His proteins. (B) Tryptic mapping of Nef-His proteins by MS. Unmodified (top panel) or TPCK-modified Nef-His proteins (bottom panel) were excised from SDS PAGE gels, digested by trypsin and injected into Micromass (Waters) for MS (MALDI-ToF). Arrows indicate the tryptic peptides containing cysteine: P1263 (C142), P1430 (C206) and P4787 (C55) from unmodified Nef-His (top panel), and P1263 (C142), P1745 (C206) and P5100 (C55) from the TPCK-modified Nef-His (bottom panel). (C) MS sequencing of the modified C-terminal peptide (P1745). Residue $10^{B}$ is the modified Cys206. Three residues Glu, Leu and Glu between Nef and His-tag are translated from the vector poly-linker region. Note, different sensitivity scales are used to show the unmodified C55 (P4787) and TPCK modified C55 (P5100). 


\section{Page 7 of 10}
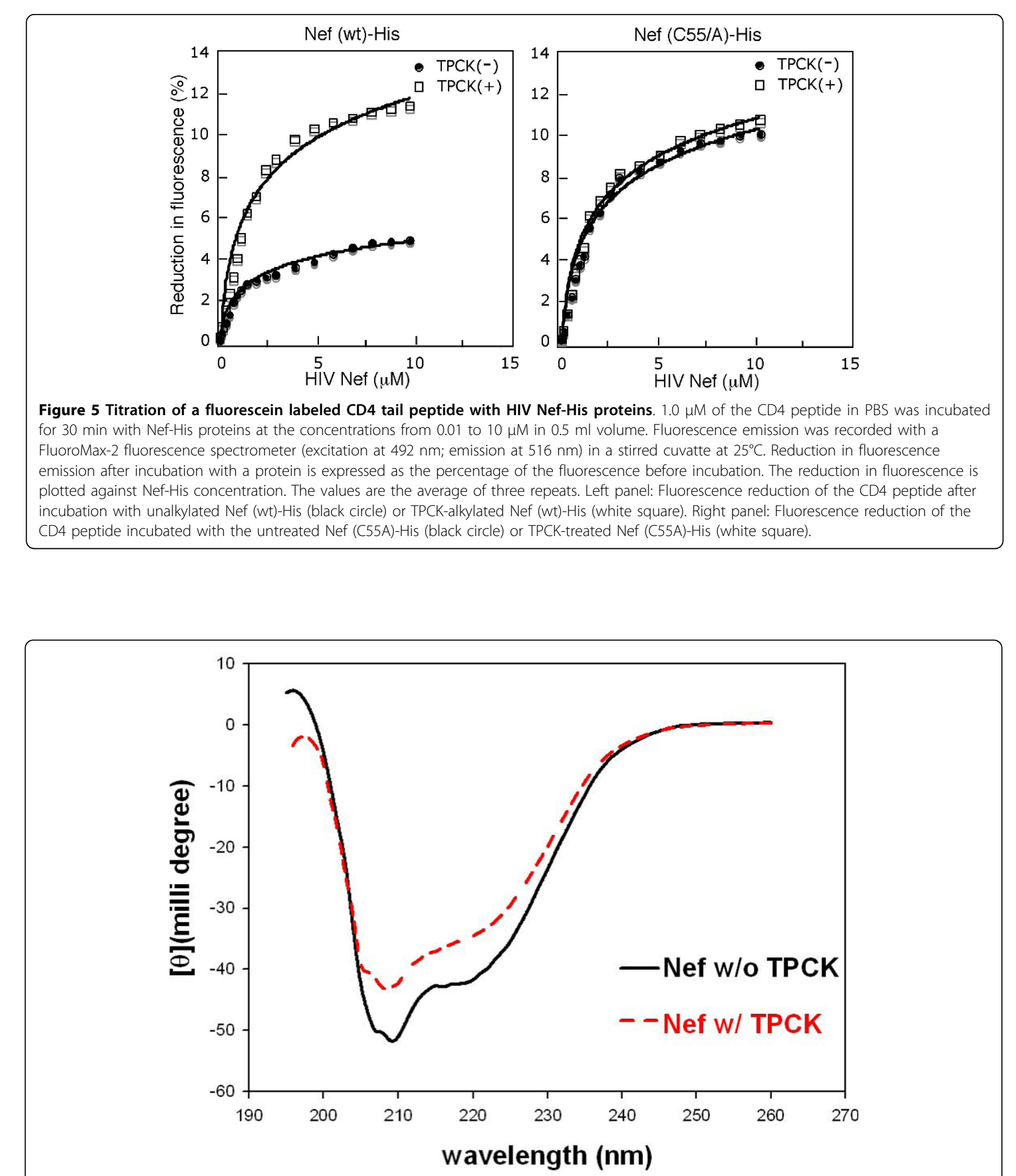

Figure 6 CD spectra of untreated Nef-His or TPCK alkylated Nef-His. The experiment was described in Methods. Samples were scanned at 260-195 nm (far-UV) at $20^{\circ} \mathrm{C}$ on an Aviv $202 \mathrm{CD}$ spectrometer (Lakewood, NJ). Acquired data were plotted using the SigmaPlot software. 
stable Nef structure upon alkylation. This is consistent with our observation that TPCK-treatment reduced Nef half-life in cultured $\mathrm{T}$ cells (not shown). Therefore, in addition to attenuation of Nef-CD4 interaction, this may be as a second mechanism for alkylation to undermine Nef's function.

\section{Discussion}

This study demonstrated that alkylation reagents, TPCK and TLCK, modify HIV-1 accessory protein Nef in live $\mathrm{T}$ cells and in vitro. Mutagenesis and MS analysis indicated that TPCK-modification of Nef is an alkylation reaction that resulted in the covalent bound of TPCK molecule to the side chains of Cys55 and Cys206 residues (Fig. 1, 2, 3, 4). Several lines of evidence suggest that the reaction is quite specific: (1) TPCK and TLCK have been used as specific serine protease inhibitors. The $\mathrm{EC}_{50}$ values of TPCK and TLCK alkylation on Nef are lower than that on chymotrypsin and trypsin, suggesting higher alkylation specificity than that of serine proteases. (2) Z-FA-FMK, a structurally very similar alkylation reagent, is inactive in modifying Nef (Fig. 1). (3) TPCK reacts with Cys but not with His residues, including those in the C-terminal His-tag, fully accessible to TPCK (Fig. 4). (4) TPCK appears to alkylate Cys55 more efficiently than to Cys206 (Fig.1).

The mechanism by which TPCK alkylates Cys residue is much less understood than the mechanism by which it alkylates His residues. It is well known that TPCK inhibits serine proteases by alkylating the His side chain at an enzyme' reactive center $[35,36]$. This understanding has rationalized the use of TPCK in signal transduction studies. In addition, some recent reports implicated the effects of alkylation at Cys, rather than at His residues [46-48]. However, how TPCK reacts with specific His or Cys is unclear. Our study showed that in case of Nef, the accessibility of Cys residues for TPCK appeared important but not sufficient for TPCK-modification. The TPCK-modified Cys55 and Cys206 are both accessible, locating in Nef N-terminal flexible region and at the $\mathrm{C}$-terminal end, respectively, whereas the none-modified C142 is buried in the Nef core [26]. However, accessibility cannot explain why TPCK did not react with any His residues despite that there are nine His residues in Nef, of which several are accessible. They include His 40 in the N-terminal flexible region and His166/His171 in the $\mathrm{C}$-terminal loop region. In addition, TPCK did not react with any His residues in the C-terminal His-tag. Probably the residues surrounding the reactive Cys or His are involved in the interaction with TPCK side chain, thus contributed to alkylation specificity.

Cys55 is next to Nef motif A56W57L58, a site important for Nef-CD4 interaction and development of AIDS [28]. The motif is also the cleavage site for HIV protease
[27]. It is conceivable that the covalent attachment of a bulky TPCK molecule to Cys55 would interfere with Nef-CD4 interaction and some other Nef functions. Fluorescence titration data indicated that TPCK-modification indeed dramatically reduced the binding strength of Nef to a CD4 tail peptide (Fig. 5). TPCK-modification may have an additional mechanism against HIV-1 by altering Nef conformation as shown by the CD spectrum change (Fig. 6) and making it unstable as suggested by a shortened half-life of Nef in T cells also (unpublished data). Unfortunately, current cell system is not fit for testing anti HIV-1 activity due to technical difficulty. TPCK only partially (50\%, maximum) alkylates wt Nef overexpressed in cultured $\mathrm{T}$ cells, leaving more than half of Nef without alkylation (Fig.1). A small fraction of unalkylated Nef protein is sufficient to downregulate CD4. Moreover, TPCK is toxic to T cells at high concentrations, which compromises the interpretation of an anti HIV-1 activity.

Our finding suggests that TPCK can serve as a prototype of a class of drugs that retains the Cys55 modification activity but has desired pharmacodynamic and pharmacological properties. A 3-D structure of the TPCK-bound Nef could guide the design and synthesis of new compounds. In this regard, we have developed a convenient method of generating large quantity of TPCK-bound Nef for structure studies (Fig. 3, 4). A comparison of such a 3-D structure with the existing 3-D model of TPCK bound to a His residue at the catalytic center of a serine protease [49] may aid the development of similar compounds that are specific for cysteine over histidine or vice versa.

\section{Conclusions}

Chloromethyl ketone reagents TPCK and TLCK directly react with Cys55 and Cys206 in Nef. TPCK alkylation at Cys55 dramatically weakens Nef-CD4 interaction, suggesting that TPCK-like small chemicals with better pharmacokinetics and pharmacodynamics may be developed for HIV disease intervention.

\section{List of abbreviations}

HIV: human immunodeficiency virus; JTAG: SV40 large T antigen-transfected human leukemic Jurkat T cells; TPCK: N- $\alpha$-p-tosyl-L-phenylalanine chloromethyl ketone; TLCK: N- $\alpha$-p-tosyl-L-lysine chloromethyl ketone; Z-FAFMK: N-CBZ-Phe-Ala fluoromethyl ketone; MHC-I: major histocompatibility complex class I.

\section{Competing interests}

The authors declare that they have no competing interests.

\section{Authors' contributions}

$\mathrm{YJ}$ is the principal investigator in this study. XZ participated in its design and helped to draft the manuscript. YC carried out the CD spectrometry study. $\mathrm{SJB}$ involved in data analysis and revision of the manuscript. All authors read and approved the final manuscript. 


\section{Acknowledgements}

We thank Tom Nubert and Chong-Feng $\mathrm{Xu}$ for the mass spectrometry. This work was supported by $\mathrm{NIH}$ grant (Al 78794) to Yong-Jiu Jin and $\mathrm{NIH}$ grant (Al 51214) to Xiaoping Zhang.

\section{Author details}

'Department of Oncological Sciences, Mount Sinai School of Medicine, New York, NY 10029, USA. ²Department of Pharmaceutics, Rutgers University, School of Pharmacy, Piscataway, NJ 08854, USA. ${ }^{3}$ Cancer Institute, Mount Sinai School of Medicine, New York, NY 10029, USA.

Received: 1 February 2010 Accepted: 26 July 2010

Published: 26 July 2010

\section{References}

1. Lundquist CA, Tobiume M, Zhou J, Unutmaz D, Aiken C: Nef-mediated downregulation of CD4 enhances human immunodeficiency virus type 1 replication in primary T lymphocytes. J Virol 2002, 76:4625-4633.

2. Benson RE, Sanfridson A, Ottinger JS, Doyle C, Cullen BR: Downregulation of cell-surface CD4 expression by simian immunodeficiency virus Nef prevents viral super infection. J Exp Med 1993, 177:1561-1566.

3. Mariani R, Skowronski J: CD4 down-regulation by nef alleles isolated from human immunodeficiency virus type 1-infected individuals. Proc Natl Acad Sci USA 1993, 90:5549-5553.

4. Lama J, Mangasarian A, Trono D: Cell-surface expression of CD4 reduces HIV-1 infectivity by blocking Env incorporation in a Nef- and Vpuinhibitable manner. Curr Biol 1999, 9:622-631.

5. Ross $T M$, Oran $A E$, Cullen BR: Inhibition of HIV-1 progeny virion release by cell-surface CD4 is relieved by expression of the viral Nef protein. Curr Biol 1999, 9:613-621.

6. Glushakova S, Munch J, Carl S, Greenough TC, Sullivan JL, Margolis L, Kirchhoff F: CD4 down-modulation by human immunodeficiency virus type 1 Nef correlates with the efficiency of viral replication and with CD4(+) T-cell depletion in human lymphoid tissue ex vivo. J Virol 2001, 75:10113-10117.

7. Cortes MJ, Wong-Staal F, Lama J: Cell surface CD4 interferes with the infectivity of HIV-1 particles released from T cells. J Biol Chem 2002, 277:1770-1779.

8. Stoddart CA, Geleziunas R, Ferrell S, Linquist-Stepps V, Moreno ME, Bare C, Xu W, Yonemoto W, Bresnahan PA, McCune JM, Greene WC: Human immunodeficiency virus type 1 Nef-mediated downregulation of CD4 correlates with Nef enhancement of viral pathogenesis. J Virol 2003, 77:2124-2133.

9. Mariani R, Kirchhoff F, Greenough TC, Sullivan JL, Desrosiers RC, Skowronski J: High frequency of defective nef alleles in a long-term survivor with nonprogressive human immunodeficiency virus type 1 infection. J Virol 1996, 70:7752-7764.

10. Lama J: The physiological relevance of CD4 receptor down-modulation during HIV infection. Curr HIV Res 2003, 1:167-184

11. Garcia JV, Miller AD: Serine phosphorylation-independent downregulation of cell-surface CD4 by nef. Nature 1991, 350:508-511.

12. Madrenas J, Schwartz RH, Germain RN: Interleukin 2 production, not the pattern of early T-cell antigen receptor-dependent tyrosine phosphorylation, controls anergy induction by both agonists and partial agonists. Proc Natl Acad Sci USA 1996, 93:9736-9741.

13. Collins KL, Chen BK, Kalams SA, Walker BD, Baltimore D: HIV-1 Nef protein protects infected primary cells against killing by cytotoxic $T$ lymphocytes. Nature 1998, 391:397-401.

14. Renkema GH, Manninen A, Mann DA, Harris M, Saksela K: Identification of the Nef-associated kinase as p21-activated kinase 2. Curr Biol 1999, 9:1407-1410.

15. Geleziunas R, Xu W, Takeda K, Ichijo H, Greene WC: HIV-1 Nef inhibits ASK1-dependent death signalling providing a potential mechanism for protecting the infected host cell. Nature 2001, 410:834-838.

16. Fackler OT, Luo W, Geyer M, Alberts AS, Peterlin BM: Activation of Vav by Nef induces cytoskeletal rearrangements and downstream effector functions. Mol Cell 1999, 3:729-739.

17. Fackler OT, Lu X, Frost JA, Geyer M, Jiang B, Luo W, Abo A, Alberts AS, Peterlin BM: p21-activated kinase 1 plays a critical role in cellular activation by Nef. Mol Cell Biol 2000, 20:2619-2627.
18. Janardhan A, Swigut T, Hill B, Myers MP, Skowronski J: HIV-1 Nef binds the DOCK2-ELMO1 complex to activate rac and inhibit lymphocyte chemotaxis. PLoS Biol 2004, 2:E6.

19. Arora VK, Fredericksen BL, Garcia JV: Nef: agent of cell subversion. Microbes Infect 2002, 4:189-199.

20. Fackler OT, Baur AS: Live and let die: Nef functions beyond HIV replication. Immunity 2002, 16:493-497.

21. Jin YJ, Cai CY, Zhang X, Burakoff SJ: Lysine 144, a ubiquitin attachment site in HIV-1 Nef, is required for Nef-mediated CD4 down-regulation. $J$ Immunol 2008, 180:7878-7886.

22. Jin YJ, Cai CY, Zhang X, Zhang HT, Hirst JA, Burakoff SJ: HIV Nef-mediated CD4 down-regulation is adaptor protein complex 2 dependent. $J$ Immunol 2005, 175:3157-3164.

23. Arold S, Franken P, Strub MP, Hoh F, Benichou S, Benarous R, Dumas C: The crystal structure of HIV-1 Nef protein bound to the Fyn kinase SH3 domain suggests a role for this complex in altered $\mathrm{T}$ cell receptor signaling. Structure 1997, 5:1361-1372.

24. Grzesiek S, Bax A, Hu JS, Kaufman J, Palmer I, Stahl SJ, Tjandra N, Wingfield PT: Refined solution structure and backbone dynamics of HIV-1 Nef. Protein Sci 1997, 6:1248-1263.

25. Lee CH, Saksela K, Mirza UA, Chait BT, Kuriyan J: Crystal structure of the conserved core of HIV-1 Nef complexed with a Src family SH3 domain. Cell 1996, 85:931-942.

26. Geyer M, Fackler OT, Peterlin BM: Structure-function relationships in HIV-1 Nef. EMBO Rep 2001, 2:580-585.

27. Freund J, Kellner R, Konvalinka J, Wolber V, Krausslich HG, Kalbitzer HR: A possible regulation of negative factor (Nef) activity of human immunodeficiency virus type 1 by the viral protease. Eur J Biochem 1994, 223:589-593.

28. Grzesiek S, Stahl SJ, Wingfield PT, Bax A: The CD4 determinant for downregulation by HIV-1 Nef directly binds to Nef. Mapping of the Nef binding surface by NMR. Biochemistry 1996, 35:10256-10261.

29. Geyer M, Munte CE, Schorr J, Kellner R, Kalbitzer HR: Structure of the anchor-domain of myristoylated and non-myristoylated HIV-1 Nef protein. J Mol Biol 1999, 289:123-138.

30. Saksela K, Cheng G, Baltimore D: Proline-rich (PxxP) motifs in HIV-1 Nef bind to SH3 domains of a subset of Src kinases and are required for the enhanced growth of Nef+ viruses but not for down-regulation of CD4. Embo J 1995, 14:484-491.

31. Xu XN, Laffert B, Screaton GR, Kraft M, Wolf D, Kolanus W, Mongkolsapay J, McMichael AJ, Baur AS: Induction of Fas ligand expression by HIV involves the interaction of Nef with the T cell receptor zeta chain. J Exp Med 1999, 189:1489-1496.

32. Bresnahan PA, Yonemoto W, Greene WC: Cutting edge: SIV Nef protein utilizes both leucine- and tyrosine-based protein sorting pathways for down-regulation of CD4. J Immunol 1999, 163:2977-2981.

33. Craig HM, Pandori MW, Guatelli JC: Interaction of HIV-1 Nef with the cellular dileucine-based sorting pathway is required for CD4 downregulation and optimal viral infectivity. Proc Natl Acad Sci USA 1998, 95:11229-11234.

34. Greenberg M, DeTulleo L, Rapoport I, Skowronski J, Kirchhausen T: A dileucine motif in HIV-1 Nef is essential for sorting into clathrin-coated pits and for downregulation of CD4. Curr Biol 1998, 8:1239-1242.

35. Schoellmann G, Shaw E: Direct evidence for the presence of histidine in the active center of chymotrypsin. Biochemistry 1963, 2:252-255.

36. Shaw $E$, Glover G: Further observations on substrate-derived chloromethyl ketones that inactivate trypsin. Arch Biochem Biophys 1970, 139:298-305.

37. Solomon DH, O'Brian CA, Weinstein IB: N-alpha-Tosyl-L-lysine chloromethyl ketone and $\mathrm{N}$-alpha-tosyl-L-phenylalanine chloromethy ketone inhibit protein kinase C. FEBS Lett 1985, 190:342-344

38. Lalou $\mathrm{Cl}$, Lederer $\mathrm{F}$ : Affinity labeling of bovine brain protein kinase $\mathrm{C}$ by tosyl lysyl chloromethane. A kinetic study. Biochimie 1993, 75:443-450.

39. Kupfer A, Gani V, Jimenez JS, Shaltiel S: Affinity labeling of the catalytic subunit of cyclic AMP-dependent protein kinase by $\mathrm{N}$ alpha-tosyl-Llysine chloromethyl ketone. Proc Natl Acad Sci USA 1979, 76:3073-3077.

40. Kinzel $\mathrm{V}$, Konig N: Interaction of protease inhibitors with the catalytic subunit of cAMP-dependent protein kinase. Biochem Biophys Res Commun 1980, 93:349-353.

41. Stoppler H, Stoppler MC, Adduci A, Koval D, Schlegel R: The serine protease inhibitors TLCK and TPCK react with the RB-binding core of 
HPV-18 E7 protein and abolish its RB-binding capability. Virology 1996, 217:542-553.

42. Fisher RJ, Koizumi S, Kondoh A, Mariano JM, Mavrothalassitis G, Bhat NK, Papas TS: Human ETS1 oncoprotein. Purification, isoforms, -SH modification, and DNA sequence-specific binding. J Biol Chem 1992, 267:17957-17965.

43. Jin YJ, Zhang X, Boursiquot JG, Burakoff SJ: CD4 phosphorylation partially reverses Nef down-regulation of CD4. J Immunol 2004, 173:5495-5500.

44. Kirchhoff F, Schindler M, Bailer N, Renkema GH, Saksela K, Knoop V, MullerTrutwin MC, Santiago ML, Bibollet-Ruche F, Dittmar MT, et al: Nef proteins from simian immunodeficiency virus-infected chimpanzees interact with p21-activated kinase 2 and modulate cell surface expression of various human receptors. J Virol 2004, 78:6864-6874.

45. Preusser A, Briese L, Baur AS, Willbold D: Direct in vitro binding of fulllength human immunodeficiency virus type 1 Nef protein to CD4 cytoplasmic domain. J Virol 2001, 75:3960-3964

46. Ha KH, Byun MS, Choi J, Jeong J, Lee K, Jue DM: N-tosyl-L-phenylalanine chloromethyl ketone inhibits NF-kappaB activation by blocking specific cysteine residues of lkappaB kinase beta and p65/RelA. Biochemistry 2009, 48:7271-7278.

47. Jitkaew S, Trebinska A, Grzybowska E, Carlsson G, Nordstrom A, Lehtio J, Frojmark AS, Dahl N, Fadeel B: N(alpha)-tosyl-L-phenylalanine chloromethyl ketone induces caspase-dependent apoptosis in transformed human $B$ cell lines with transcriptional down-regulation of anti-apoptotic HS1-associated protein X-1. J Biol Chem 2009, 284:27827-27837.

48. Heussler VT, Fernandez PC, Machado J Jr, Botteron C, Dobbelaere DA: Nacetylcysteine blocks apoptosis induced by $\mathrm{N}$-alpha-tosyl-Lphenylalanine chloromethyl ketone in transformed T-cells. Cell Death Differ 1999, 6:342-350.

49. Mac Sweeney A, Birrane G, Walsh MA, O'Connell T, Malthouse JP, Higgins TM: Crystal structure of delta-chymotrypsin bound to a peptidyl chloromethyl ketone inhibitor. Acta Crystallogr D Biol Crystallogr 2000, 56:280-286.

doi:10.1186/1742-6405-7-26

Cite this article as: Jin et al: Alkylating HIV-1 Nef - a potential way of HIV intervention. AIDS Research and Therapy 2010 7:26.

\section{Submit your next manuscript to BioMed Central and take full advantage of:}

- Convenient online submission

- Thorough peer review

- No space constraints or color figure charges

- Immediate publication on acceptance

- Inclusion in PubMed, CAS, Scopus and Google Scholar

- Research which is freely available for redistribution

Submit your manuscript at www.biomedcentral.com/submit
Biomed Central 